\title{
Relationship Between Psychodemograhic Factors And Perceived Stigmatization Among People Living With Hiv/Aids In Ibadan, Nigeria
}

\author{
Olalekan T. Kazeem \\ Department of Psychology, University of Ibadan, Nigeria
}

\begin{abstract}
The issue of perceived stigmatization among PLWHAs remains a major hindrance in the prevention and post-exposure control of HIV/AIDS. The relationship between HIV/AIDS cognition (HAC), HIV selfdisclosure (HSD) and perceived stigmatization (PS) was investigated. The influence of age and gender on PS was also examined. PLWHA $(N=421)$ in Ibadan, Nigeria were purposively selected to participate in the crosssectional study, using a 70-item structured self report questionnaire. A positive relationship of HAC $(r=.10, d f$ $=421, P<.05)$ and HSD $(r=.60, d f=421, P<.05)$ with PS was observed. A $2 \times 2 \times 2 \times 2$ factorial analysis showed that young females with poor $H A C$, but high on $H S D(n=38,=106.57, S D=4.8)$ were most likely to experience Perceived stigmatization. HAC and HSD jointly predicted PS $\left(R^{2}=.36 ; F(2,418)=120.29 ; P<.05\right)$. Female PLWHA experience PS more than males $(t(419)=-4.40 ; P<.05)$. Age had no significant influence on PS. This establishes that a relationship exist between HAC, HSD, and PS. Policies and actions should therefore focus on these issues in HIV prevention, care and support.
\end{abstract}

Key words: HIV self-disclosure, Stigmatization, psychosocial factors, HIV self-disclosure, Nigeria.

\section{Background}

In Nigeria, as elsewhere, the Acquired Immune Deficiency Syndrome (AIDS) is perceived as a disease of "others"- of people living on the margins of society, whose lifestyles are considered "perverted" and "sinful". Stigma is a term that involves both deviance and prejudice but goes beyond both. Stigma involves perceptions of deviance but extends to more general attributions about character and identity. Stigma is more inclusive than prejudice because it involves individual-based responses to deviance, as well as group-based reactions as a function of category membership. Because stigma is socially defined, there is a considerable variation across cultures (Dovidio, Majo and Crocker, 2006). Stigmatization, discrimination and denial are the expected outcomes of such values, affecting life in families, communities, workplaces, schools and health care settings. People living with HIV and AIDS (PLWHA) continue to be burdened by poor care and inadequate services, while those with the power to help do little to make the situation better (Mark and Richardson, 2005).

An estimated 4.6 percent of the population are living with HIV and AIDS in Nigeria (UNGASS, 2010). Although HIV prevalence is much lower in Nigeria than in other African countries such as South Africa and Zambia, the size of Nigeria's population (around 149 million) meant that by the end of 2009, there were about 2.98 million people living with HIV (UNGASS, 2010). AIDS is not only claiming so many lives, about 192,000 in 2009, Nigeria's life expectancy has declined significantly. The average life expectancy was 54 years for women and 53 years for men in 1991, (UNGASS, 2010). However, in 2009 these figures had fallen to 48 for women and 46 for men (CIA World Fact book, 2010).

A plethora of quantitative studies confirm that HIV-related stigma and discrimination are highly prevalent and feature prominently in the lives of people living with HIV in settings with epidemics as diverse as the United States, Vietnam, and South Africa. Comparison of prevalence across region or sample is complicated by the differences among stigmatizing incidents inquired about as well as different time-frames. The experience of stigma was universal among a small sample in Brazil, and nearly universal among a sample of women in Vietnam (Melchior and Brickley, 2008). One study in the US indicated that 8 out of 10 families experienced discrimination (Cusick, Rhodes and Bogart, 2008). In contrast, another study of women with HIV in the US found the prevalence of stigma to be lower, at around $17 \%$ of the sample (Wingood, 2007).

A large household-based study in Kenya found that $75 \%$ of HIV-positive respondents had experienced "perceived stigma" (Odindo, Hudson and Mwanthi, 2008). Slightly more than one-half of respondents experienced "perceived stigma" in a China sample ( $\mathrm{Li}$, Cao, Wu and Xiao, 2007) as had one-third of respondents in South India (Subramanian, Gupte, Dorairaj, Periannan and Mathai, 2009). In the Dominican Republic, a study highlighted the importance of "verbal stigma", with more than $60 \%$ reporting being gossiped about and between $25-30 \%$ reporting verbal abuse. Furthermore, the study found striking gender differences, with women experiencing more of nearly every form of "perceived stigma"- a pattern found in earlier work in Tanzania - and gender-based violence emerging as a prominent concern as well (MacQuarrie, Nyblade, Philip, 
Kwesigabo and Mbwambo, 2006). Perceived stigma was a predictor for declining to test for HIV among both men and women in a study of Nigerian youth and adults in South Africa (Hutchinson and Mahlalela, 2006).

Stigma and discrimination continue to affect those living with and affected by HIV disease as well as their health care providers. It has also become a substantial barrier to accessing primary and secondary HIV and AIDS care and prevention services (Rao, Kekwaletswe, Hosek, Martinez and Rodriguez, 2007).It is a barrier to voluntary testing and counseling and thus may result in delays in accessing care and treatments, and consequently increase illness and death.

Holzemer, Hudson, Kirksey, Hamilton and Bakken (2001) developed a conceptual model of perceived HIV/AIDS-related stigma, based on data from focus groups with 251 people. In the model, HIV/AIDS stigma is described as a process, with four dimensions: triggers, stigmatizing behaviours, type of stigma, and outcomes of stigma. The outcomes of stigma-for example poor health- may also serve as triggers, starting the process again. The stigma process occurs within the environment (political, social, etc), and in the context of the health care system and the individuals who may enact stigma. Non disclosure was identified as one of the outcomes of HIV stigma. It is therefore necessary to investigate relationship between psychological factors and perceived stigmatization for sub Sahara Africa.

HIV self disclosure is the tendency of an individual to be free to autonomously discuss his/her HIV status with a sexual partner, family, or units of a society. Unfortunately, the potential for rejection, abandonment, physical and emotional abuse and other adverse consequences create substantial barriers to disclosing HIV status (Rothenberg and Paskey, 1995). HIV disclosure is a complex issue since quite often disclosure of status is also linked to disclosure of other clandestine behaviours (such as same sex activity, injection drug use, or any HIV risk behaviour (Adejumo, 2004).

In addition, significant disincentives and barriers to revealing one's HIV diagnosis persist. These include fears of rejection and abandonment, discriminating treatments such as eviction or termination of employment, retribution, violence, and other forms of abuse. Most of these possible outcomes are based on the social stigma that is widely acknowledged to be associated with an HIV diagnosis. Additionally, divulging that one is HIV -infected may expose other stigmatized behaviours or identities (Davidio and Richardson, 2005).

Data throughout the world suggests that failure to disclose HIV status is related to three major problems. First, substantial numbers of new HIV infections could occur among partners of HIV-positive persons who do not disclose their status (Mohammed, Kissinger and Barnes, 2007). Second it could also hinder access to anteretroviral therapy (Ogundahunsi and Sullivan, 2005). Third, failure to disclose could hinder social support required for mental health (Derlega, Metts, Petronio and Margulis, 1993). These problems emanate from stigma related to HIV/AIDS.

Cognitive representations of social objects are referred to as schemas. These schemas are a mental structure that represents some aspects of the world. It is generally accepted that positive HIV cognitive representation can result in better mental and physical health (Vaux, Derlega, Barbee, Sachdev, Antle and Greene, 2002), protecting migrants from adaptation stress and improving coping abilities (Folkman and Moskowitz, 2000). A clear link has also been shown between positive HIV cognition, low risk sexual behaviour and disclosure of HIV status (Reilly and Woo, 2004). Disclosure is considered good for mental and physical health as it can increase positive HIV cognition, decrease anxiety and depression, and reduce isolation and high risk sexual behaviour (Cusick, Rhodes 1999; Reilly and Woo 2004; Serovich, 2005). However, the role of HIV/AIDS cognition and HIV disclosure one hand and perceived stigmatization have not been adequately documented in literature.

The goal of this study is to assess the relationship between perceived stigmatization among PLWHA and psychological factors namely; HIV disclosure and HIV cognition. Specifically, the objectives of the study are to:

i. Discover if psychological factors (HIV disclosure and HIV cognition) will have any relationship with perceived stigmatization among PLWHA and

ii. Examine if there will be differences in the factorial combinations of the influences of psychosocial factors (age and gender) on perceived stigmatization.

\section{Method}

This correlational study adopted a cross-sectional design. The independent variables were HIV self disclosure and HIV cognition. The dependent variable was perceived stigmatization. This design was also used in a similar study conducted by Rubin, D. L. (2007).

The study was conducted in four centres for PLWHA in Ibadan, Oyo State, Nigeria. The centres were; Adeoyo Maternity Hospital, Ibadan, APIN/PEPFAR units at St. Mary Hospital, Eleta, Ibadan, Family Health and Population Action Council, Ibadan, and Association for Reproductive and Family Health (AFRH), Ibadan. Ibadan city. These sites were selected because of their focus and involvement in caring for PLWHA. 
PLWHA in the centres participated. Prospective participants were required to fill an inclusion-exclusion criteria including:

1. Diagnosed as HIV positive before the study

2. Currently registered with any of the four centres caring for PLWHA in Ibadan

3. English literate and

4. Personally willing to participate after an informed consent process

Even though about 765 PLWHA were fully registered at the Centres, only about 500 of them fulfilled the inclusion criteria, and were purposively included as participants. Of these,

421 questionnaires were correctly filled and returned, representing $84.2 \%$ response rate. These were made up of 278 (66\%) females and $143(34 \%)$ males. The participants' age ranged between 23 and 52 years with a mean age of 34 years.

\section{Instruments}

Data was collected with the use of a 70-item self-report questionnaire made up of five sections. The 8item Section A was designed to tap information about respondents' socio-demographic characteristics. Such information included: gender, age, marital status, religion, HIV disclosure status, educational background, and vocation/profession. Section B had 10 items. It contained the HIV self disclosure scale developed by Kalichman and Nachimson (1999). The scale is designed in a Likert format with responses ranging between strongly agree and strongly disagree. Kalichman (2000) reported a Cronbach alpha of .85, but a re-validation during this study yielded a Cronbach alpha of .79. The norms established during this study was $(\mathrm{N}=421, \overline{\mathrm{X}}=28.6, \mathrm{SD}=13.5)$. The higher the score on the scale, the higher disclosure rate, and vice versa.

Section C of the questionnaire developed by Berger, Ferrans and Lashley (2001) had 40 items designed to obtain information on HIV perceived stigmatization. The self-report Likert-type instrument has four graduated responses ranging from strongly agree to strongly disagree. It had a reliability coefficient of .96; a re-validation yielded an alpha coefficient of .79. The mean obtained was $(\mathrm{N}=421, \bar{X}=99.2, \mathrm{SD}=4.8)$. Any score below the mean implies low perceived HIV stigmatization.

Section D was a 12 -item self report scale developed by Dr. D. Shah to measure HIV cognition among PLWHA. It has a 5-point graded responses ranging from "had thought very frequently" to "had thought very occasionally". The developer reported a reliability coefficient of .91. A re-validation during this study yielded an internal coefficient of .90. Any score above the mean and standard deviation $(\mathrm{N}=421, \overline{\mathrm{X}}=38.2, \mathrm{SD}=20.5)$ is interpreted as good HIV cognition, vice versa (Shah, Thornton and Burgess, 1997).

\section{Data Collection}

The researcher obtained permission from the Department of Psychology, University of Ibadan, to conduct the study. Being a vulnerable group, the researcher thereafter sent the protocol for further institutional review and ethical approval from the State Ministry of Health Research Ethics Committee, which was granted following due ethical review process. The researcher also discussed the protocol with the management of the selected hospitals. Each of the Centres' management ascertained that the research is of minimal psychological or physical harm (if any at all); the respective institutions thereafter gave permission to conduct the study.

At the centres, the medical records of the participants were reviewed to identify potential participants that meet the inclusion-exclusion criteria. While the participants waited at the clinics to see their physicians, the researcher discussed the purpose of the study and gave the questionnaires to prospective participants, including a detailed informed consent document. Only willing and consenting PLWHA in attendance at the clinics were recruited as research participants. They were allowed to read the questionnaire and respond accordingly. This took an average of 30 minutes.

A total of 500 of them fulfilled the inclusion criteria in the four Centres, and were purposively included as participants. Of these, only 421 questionnaires were correctly and completely filled. Completed questionnaires were sorted, coded, and entered into the Statistical Package for Social Sciences for data analysis. 
Relationship Between Psychodemograhic Factors And Perceived Stigmatization Among People

\section{Results}

Table 1: Summary of $2 \times 2 \times 2 \times 2$ factorial levels of gender, age, HIV cognition and HIV disclosure on perceived stigmatization

\begin{tabular}{|l|l|l|l|l|l|c|c|}
\hline Gender & Age & $\begin{array}{c}\text { HIV } \\
\text { cognition }\end{array}$ & $\begin{array}{c}\text { HIV } \\
\text { disclosure }\end{array}$ & Interaction & \multicolumn{3}{l|}{ Perceived stigmatization } \\
on Xnk \\
\hline
\end{tabular}

Key: $\mathrm{F}=$ Female, $\mathrm{M}=$ Male, $\mathrm{Y}=$ Young, $\mathrm{O}=\mathrm{Old}$, $\mathrm{P}=$ Poor HIV Cognition, $\mathrm{G}=$ Good HIV Cognition

L=Low HIV disclosure $\mathrm{H}=$ High HIV disclosure.

Table 1 revealed the influence of gender, age, HIV self disclosure and HIV cognition on perceived stigmatization, among PLWHA in this study. It could be observed that those who were females, young, poor on HIV cognition, but with high HIV disclosure recorded the highest mean $(n=38, \bar{X}=106.57, \mathrm{SD}=4.8)$ on perceived stigmatization. This means that PLWHA with these attributes are most likely to experience stigmatization compared to PLWHA with other characteristics as seen. The group with the second most likely to experience stigma are those who are females, old, with good HIV cognition and high on disclosure $(\mathrm{n}=59$, $\mathrm{X}=105.76, \mathrm{SD}=5.5$ ). Female respondents who are old, poor HIV cognition, but with high on HIV disclosure $(\mathrm{n}=50, \overline{\mathrm{X}}=105.14, \mathrm{SD}=5.5)$ ranked third on perceived stigmatization, while young females with good HIV cognition and high HIV disclosure $(\mathrm{n}=33, \overline{\mathrm{X}}=104.9, \mathrm{SD}=5.4)$ ranked fourth.

The group with the fifth rank in HIV disclosure are young males with good HIV cognition and high score on HIV disclosure $(\mathrm{n}=15, \overline{\mathrm{X}}=103.4, \mathrm{SD}=3.9)$. Young males, with poor HIV cognition but high HIV disclosure ranked sixth $(\mathrm{n}=18, \overline{\mathrm{X}}=103.38, \mathrm{SD}=3.5)$ while old males with good HIV cognition and high HIV disclosure were in seventh position $(\mathrm{n}=15, \overline{\mathrm{X}}=102.53, \mathrm{SD}=7.1)$. Old males with poor HIV cognition but high HIV disclosure ( $\mathrm{n}=21, \overline{\mathrm{X}}=102.42, \mathrm{SD}=4.9)$ ranked eighth in perceived stigmatization. Old males with good HIV cognition but low HIV disclosure $(\mathrm{n}=17, \overline{\mathrm{X}}=101.76, \mathrm{SD}=3.9)$ ranked ninth, while young females with poor HIV cognition and low disclosure $(\mathrm{n}=13, \mathrm{X}=100.3, \mathrm{SD}=3.7)$ ranked tenth.

Old females with poor HIV cognition, low HIV disclosure $(n=35, \bar{X}=100.2, S D=3.5)$ ranked eleventh, while young females with poor HIV cognition and low disclosure $(n=21, \bar{X}=100.19, \mathrm{SD}=3.8)$ ranked twelfth. Old female PLWHA with good HIV cognition but low disclosure ranked thirteenth in perceived stigmatization $(\mathrm{n}=29, \overline{\mathrm{X}}=99.24, \mathrm{SD}=4.1)$, while young male PLWHA with poor HIV cognition and low disclosure ranked fourteenth $(\mathrm{n}=16, \overline{\mathrm{X}}=99.12, \mathrm{SD}=4.1)$. Old male PLWHA with poor HIV cognition and low disclosure ranked fifteenth ( $n=23, \bar{X}=98.65, S D=4.6)$. The group with the least likelihood of experiencing stigmatization include young male PLWHA with good HIV cognition and low disclosure $(\mathrm{n}=13, \overline{\mathrm{X}}=98.53, \mathrm{SD}=4.7)$.

Table 2: Means, standard deviation and Pearson Product Moment Correlation among psychological variables and perceived stigmatization

\begin{tabular}{llcccc}
\hline & Means & SD & 1 & 2 & 3 \\
\hline Perceived stigmatization & 102.8 & 5.4 & 1 & & \\
\hline HIV Cognition & 35.5 & 5.5 & .10 & 1 & -.01 \\
\hline HIV self disclosure & 26.1 & 3.4 & $.60 * *$ & 1
\end{tabular}

* $\mathrm{P}<.05$

Table 2 shows that HIV self disclosure had strongest positive significant relationship with perceived stigmatization $(\mathrm{r}=.60, \mathrm{df}=420 ; \mathrm{P}<.05)$. This implies that as PLWHA disclose their status, stigmatization increases and vice versa.HIV cognition also had a weak positive relationship with perceived stigmatization $(r=.10, d f=420$; $\mathrm{P}<.05)$. 
Relationship Between Psychodemograhic Factors And Perceived Stigmatization Among People

Table 3: Multiple regression summary table showing independent and joint influence of psychological variables on perceived stigmatization

\begin{tabular}{|c|c|c|c|c|c|c|c|c|c|}
\hline \multicolumn{4}{|c|}{ Independent effect s } & \multicolumn{6}{|c|}{ Joint effects } \\
\hline Yariables.mm & B & E. & Beta & $\mathbf{T}$ & & $\mathbf{R}$ & $\mathbf{R}^{2} \mathbf{A}$ & $\operatorname{ldj} \cdot \mathbf{R}^{2}$ & $\mathbf{P}$ \\
\hline HIV Cognition & .102 & .039 & .103 & 2.65 & $<.05$ & .604 & .365 & .362 & $<.05$ \\
\hline HIV self disclos & .936 & .061 & .597 & 15.31 & $<.05$ & & & & \\
\hline
\end{tabular}

Table 3 shows that HIV cognition and HIV self disclosure jointly predicted perceived stigmatization $\left(\mathrm{R}^{2}=.36 ; \mathrm{F}(2,418)=120.29 ; \mathrm{P}<.05\right)$. The variables jointly accounted for 36.2 percent $\left(\mathrm{Adj} . \mathrm{R}^{2}=0.362\right)$ variance in the perceived stigmatization among people living with HIV/AIDS. In term of independent effect of each variable, HIV self disclosure had most potent prediction $(\beta=0.597, \mathrm{t}=15.31, \mathrm{P}<.05)$. The variable accounted for 59.7 percent $(\beta=0.597)$ variance in perceived stigmatization among PLWHA. The Beta also implies that as HIV disclosure increases; perceived stigmatization increases and vice versa. HIV cognition also predicted perceived stigmatization $(\beta=0.103, \mathrm{t}=2.65, \mathrm{P}<.05)$.It accounted for 10.3 percent $(\beta=0.103)$ variance.

Table 4: Summary of t-test of independent showing mean difference between male and female on perceived stigmatization

\begin{tabular}{|l|l|l|l|l|l|l|}
\hline Gender & $\mathrm{N}$ & $\overline{\mathrm{X}}$ & SD & Df & T & $\mathrm{P}$ \\
\hline Male & 143 & 101.25 & 4.98 & 419 & -4.40 & $<.05$ \\
\hline Female & 278 & 103.60 & 5.56 & & & \\
\hline
\end{tabular}

Table 4 revealed that there was significant difference in male and female on perceived stigmatization ( $\mathrm{t}$ $(419)=-4.40 ; \mathrm{P},<.05)$. This implies that female PLWHA experience perceived stigmatization $(\overline{\mathrm{X}}=103.6)$ more than male $(\overline{\mathrm{X}}=101.25)$.

Table 5: Summary of t-test of independent showing mean difference between young and old on perceived stigmatization

\begin{tabular}{|l|l|l|l|l|l|l|}
\hline Age & $\mathrm{N}$ & $\overline{\mathrm{X}}$ & SD & Df & T & $\mathrm{P}$ \\
\hline Young & 167 & 102.9 & 5.2 & 419 & .55 & $>.05$ \\
\cline { 1 - 5 } Old & 254 & 102.6 & 5.6 & & & \\
\hline
\end{tabular}

Table 5 results indicate that young and old were not significantly different on mean score on perceived stigmatization $(\mathrm{t}(419)=.55, \mathrm{p}>.05)$, While the mean score of young PLWHA was $\overline{\mathrm{X}}=102.9$, old PLWHA had a mean score of $\bar{X}=102.6$ in the study.

\section{Discussion}

The results from the present study showed that HIV cognition and HIV self disclosure had significant positive relationship with perceived stigmatization but HIV self disclosure was more related. Old were not significantly different from young in perceived stigmatization, but female PLWHA experience more stigmatization than male PLWHA. It was also discovered that young females with poor on HIV cognition, but high HIV disclosure mostly experience perceived stigmatization followed by older females with good HIV cognition and high disclosure. On the other hand, individuals that are most unlikely to experience perceived stigmatization are old male PLWHA with poor HIV cognition and low HIV disclosure, followed by young male PLWHA with poor HIV cognition and low HIV disclosure.

In agreement with earlier findings (Olley, Seedat, and Stein, 2004) people who believe that PLWHA are stigmatized in a society are very unlikely to disclose their HIV status to either their sex partners, employer, or members of their society. People living with HIV/AIDS that disclosure experience more stigmatization than those who did not, as the study found a positive significant relationship between HIV self disclosure and perceived stigmatization. Going by this, information about HIV infection is believed to be private and confidential; which if revealed, could lead to rejection and stigmatization (Rothenberg and Paskey, 1995). Yet when HIV status is not disclosed to sexual partners, safer sexual practices may not be followed, and further spread of the disease may thereby result. This may be further explained by failure of many governments to legislate and enforce the protection of PLWHA in work places where co-workers and employers often discriminate against PLWHA. In many other cases employers often feel reluctant to engage PLWHA. Even when already actively employed in an organisation, employers often sack staff known to be HIV positive either as a result of the fear of infecting fellow employees or fear of reduced productivity. In some other societies, 
employers may also have the fear that consumer satisfaction and patronage may reduce drastically if consumers or members of the society discover that an organization's services were provided by PLWHA.

The relatedness of HIV cognition in perceived stigmatization in this study might be due to the connectedness between cognition, perception, reasoning and knowledge. Elwood and Carte (2003) had earlier found that perception of illness influence individual health behaviour, especially attitude and tendency to seek health care. It is also in agreement with the finding of Ciccarone ,Blance and Babalola(2003); as well as Marks and Crepaz(2001) where they discovered that of men who are HIV-positive with positive cognition, only about half of them disclose their serostatus to sexual partners before engaging in unprotected intercourse. An individual's mental reasoning or perception about a specific situation is more likely to influence the individual's mental evaluation, attitude, and behaviour related to the event. This means that if an individual sees HIV infection as challenging but surmountable; with a basic understanding of the aetiology, course, treatment and prognosis of the infection, such an individual is likely to make a meaningful cost-benefit analysis of HIV disclosure and stigmatization. With this such an individual will make sound decision based on adequate information and personal motivation to disclose HIV status irrespective of the challenges.

Males were significantly different from females in perceived stigmatization, but there was no significant different between old and young PLWHA. The above finding further explains the wide variations in gender role and gender awareness in many parts of Africa. In south west Nigeria where this study took place, there is significant gender difference in PLWHA tendency to experience stigmatization. However, whether male or female the society discriminates against PLWHA, hence the difference in the participants' stance on HIV perceived stigmatization.

\section{Conclusion}

One of the major contributions of this study to HIV/AIDS literature is that factorial combination of certain psychological and socio-demographic factors yield differences in perceived stigmatization. For instance, it was discovered that young females with poor HIV cognition but high HIV disclosure were most likely to experience perceived stigmatization. This implies that PLWHA with these characteristics are more likely to battle with stigmatization. It also further confirms the finding that HIV cognition and HIV self disclosure are critical in predicting perceived stigmatization. Female PLWHA experience stigmatization more than male probably because of inherent emotional disposition, experience and sexual activity. On the other hand, young male PLWHA with poor HIV cognition and low HIV disclosure are most unlikely to experience stigmatization because of perceived emotional insecurity, immaturity, and fear of rejection.

It could therefore be concluded that good HIV cognition and high self disclosure enhance perceived stigmatization among PLWHA. These factors as well as gender are related to perceived stigmatization. Serovich(2001) also found that fears of rejection and abandonment, discriminating treatment such as eviction or termination of employment, retribution, violence, and other forms of abuse are significant disincentives and barriers to revealing one's HIV diagnosis persist.

HIV non disclosure remains an enormous barrier to the fight against HIV and AIDS. The lesson learnt here is that social stigma in term of prejudice, discrimination and other forms of stigmatization are major barriers to HIV disclosure. A re-focusing of HIV/AIDS prevention programmes on these factors by policy relevant policy makers, programme planners in governmental and non-governmental organisations is critical not only for HIV prevention, but the care and support of PLWHA.

\section{References}

Adejumo, A.O. (2004). The influence of psychosocial factors and the effectiveness of behavioural techniques on HIV sexual risk behaviour among adolescents in Ibadan, Nigeria. Unpublished doctorate thesis of the University of Ibadan, Ibadan, Nigeria.pp. 211-231.

[2] Berger, B. E., Ferrans, C. E., Lashley, F.R. (2001). "Measuring stigma in people with HIV: Psychometric assessment of the HIV stigma scale," Research in Nursing and Health 24: 518-529.

[3] Central Intelligence Agency (2010). World Fact Book: Nigeria. Downloaded on June 10, 2010. Available at https://www.cia.gov/library/publications/the-world-factbook/geos/ni.html.

[4] Ciccarone, D.H., Blance, I.A. and Babalola F.A. (2003). Self-disclosure Experience of People with HIV Infection in Dedicated and Mainstreamed Dental Facilities. Journal of Public Health Dentistry, 56 (4), pp 223-225.

[5] Cusick, K., Rhodes M; Reilly, B., Woo, T. and Serovich, H. (2001).Sex without disclosure of positive HIV serostatus in a US probability sample of persons receiving medical care for HIV infection. American Journal of Public Health, 93(6):949-954.

[6] Dovidio K., Majo H. and Crocker M.(2006). Experiences of Stigma and Access to HAART in Children and Adolescents Living with HIV/AIDS in Brazil." Social Science \&Medicine 62(5): 1219-1228.

[7] Degefa, A., Sanders, E. J., Mekonnen, Y., Messele, T., Wolday D., Dorigo-Zetsma, W., Mekonnen W., Schaap, A. and Dukers, N.H. (2003). Knowledge and attitude towards anti retroviral therapy among factory workers participating in a cohort on HIV and AIDS, Addis Ababa, Ethiopia. Ethiopian Medical Journal, 41 (Supply 1) :75-87.

[8] Derlega, V. J., Metts, S., Petronio, S. and Margulis, S. T. (1993). Self-disclosure Newbury Park, CA: Sage.

[9] Elwood, S. and Carter, N. (2003). Egypt In-Depth Study on the Reasons for Nonuse of Family Planning: Results of a Panel Survey in Upper Egypt. Calverton, MD: NationalPopulation Council (Egypt) and Macro International. 
[10] Folkman A.S. and Moskowitz, D.B. (2000).Conflicting messages: how criminal HIV disclosure Laws undermine public health efforts to control the spread of HIV.AIDS and Behaviour. 10: 451-461.

[11] Herek, G.M. and Glunt, E.K. (1988). An epidemic of stigma: Public reactions to AIDS

[12] American Psychologist, 43(11), 886- 891.

[13] Hennessy, R. (2010). Safe Sex and HIV: A Clinical Perspective. The Albion Street Centre, Sydney NSW. Downloaded on August 10, 2010 via www.hivhepsti.info/ documents/RuthHennessy.ppt

[14] Holzemer, W.L., Hudson, A., Kirksey,K. M.,Hamilton,M.J. and Bakken.S.(2001).The revised sign and symptom check-list for HIV (SSC-HIV rev).Journal of the association of Nurses in AIDS Care 12(5),60-70.

[15] HolzemerW.L.,Uys,L.,Makoae,L.,Stewart,A.,Phetlhu,R.,Dlamini,P.S.,Greeff,M.,Kohi,T.W.,Chirwa, M., Cuca,Y. and Naidoo,J.(2007). A conceptual model of HIV/AIDS stigma from five African countries. Journal of Advanced Nursing 58(6), 541551 .

[16] Hutchinson, P. L .and Mahlalela X. (2006). "Utilization of voluntary counseling and testing services in the Eastern Cape, South Africa." AIDS Care 18: 446-455.

[17] Li, L., Cao, H., Wuo, S. and Xiao, L.(2007). "Diffusion of Positive Aids Care Messages Among Service Providers in China." AIDS Education and Prevention_19(6): 511-518.

[18] Kalichman, S.C. and Nachimson, D. (1999). Self-Efficacy and Disclosure of HIV-Positive Serostatus to Sex Partners. Health Psychology; 18:281-287.

[19] Kalichman, S.C. (2000). HIV transmission risk behaviors of men and women living with HIV-AIDS: prevalence, predictors, and emerging clinical interventions. Clinical Psychology: Science Practice. 2000; 7:32-47.

[20] MacQuarrie, K., L., Nyblade, F., Philip, G., Kwesigabo O. and Mbwambo J. (2006).Gender Differences and the Experience of Stigma (Abstract No. THPE0618). AIDS 2006, XVI International AIDS Conference, Toronto, Canada.

[21] Marks, G., Richardson, J. L. and Maldonado, N. (2005). Self-disclosure of HIV infection to sexual partners. American Journal of Public Health. 81:1321-1322.

[22] Marks, G. and Crepaz, N. (2001). HIV-positive men's sexual practices in the context of self-disclosure of HIV status. Journal of Acquired Immune Deficiency Syndrome, 27:79

[23] Melchior, A. and Brickley, R.(2008). Are HIV care providers talking with patients about safer sex and disclosure? A multi-clinic assessment. AIDS.; 16:1953-1957.

[24] Mohammed, H. and Kissinger, P. (2006). Disclosure of HIV Serostatus to Sex Partners in Rural Louisiana, AIDS Care, Vol. 18, Issue S1, pp. 62-69.

[25] Niccolai, L. M., King, E., D'entremont, D.and Pritchett, E. N. (2006). Disclosure of HIV Serostatus to Sex Partners: A New Approach to Measurement. Sexually Transmitted Diseases, 33:102-105.

[26] Odindo, M. A. and Mwanthi A.M. (2008). "Role of Governmental and Non-Governmental Organizations in Mitigation of Stigma and Discrimination Among HIV/AIDS Persons in Kibera, Kenya." East Africa Journal of Public Health_5(1): 1-5.

[27] Ogundahunsi, O. A., Daniel, O.J. and Oladapo, O.T. (2008). Adherence to antiretroviral drugs among AIDS patients in Sagamu, Nigeria. International Journal of Biomedical and Health Sciences, (2), 4.

[28] Olley, B. O., Seedat, S. and Stein, D. J. (2004). Self-Disclosure of HIV serostatus in Recently Diagnosed Patients with HIV in South Africa. African Journal of Reproductive Health 2004, 8:71-76.

[29] Pennebaker, J. W., Colder, M. and Sharp, L.K. (1990). Accelerating the coping process.Journal of Personality and Social Psychology, 58, 528-537.

[30] Perry, S. W., Card, C. A. L., Moffat, M., Ashma, T., Fishman, B. and Jocobsberg, L. B.(1994). Self disclosure of HIV infection to sexual partners after repeated counselling. AIDS Education and Prevention. 6: 403-411.

[31] Rao, D., Kekwaletswe, T.C., Hosek,S., Martinez,J. and Rodriguez,F.,(2007).Stigma and social barriers to medication adherence with urban youth living with HIV/AIDS care 19(1),28-33

[32] Rao, B.N., Gupta, G. and Weiss, E. (1993). "Women's Lives and Sex: Implications for AIDS

[33] Prevention." Culture, Medicine and Psychiatry 17.

[34] Riley, B.A. and Woo I. (2004). "Gender, power, and population change." Population Bulletin 52(1). Washington, DC: Population Reference Bureau.

[35] Rothenberg, K H. and Paskey, S. J. (1995). The risk of domestic violence and women with HIV infection: implications for partner notification, public policy, and the law.American Journal of Public Health, Vol. 85, Issue 11 1569-1576.

[36] Rubin, D. L. (2007). Factors Leading to Self Disclosure of a Positive HIV Diagnosis in Nairobi. Qualitative Health Research, Vol. 17, No. 5, 586-598.

[37] Serovich, J. M. (2001). A test of two HIV disclosure theories. AIDS Education Prevention, 13 (4) $335-364$.

[38] Shah, D., Thornton, S.and Burgess, P. (1997). Sexual risk cognitions questionnaire: a reliability and validity study. AIDS Care, 9, $471-480$.

[39] Simon, C. E., Bowles, D. D., King, S. W. and Roff, L. L. (2004). Mentoring in the careers of African American women in social work education. Affiliated, 19 (2), 134-145.

[40] Skogmar, S, Shakely, D and Lans, M.(2006). Effect of antiretroviral treatment and counseling on disclosure of HIV-serostatus in Johannesburg, South Africa. AIDS Care; 18:725-730.

[41] Stein, M. D., Freedberg, K. A., Sullivan, L. M., Savetsysky, J. M., Levenson, S. M., Hingson, R. and Samet, J. H.(2003). Sexual Ethics: Disclosure of HIV sero status to partners. Archives of Internal Medicine; 158 (9), 253-257.

[42] Subramanian, T., Gupte, V. S., Dorairaj, V., Periannan M. and Mathai A.K. (2009)."Psycho-social impact and quality of life of people living with HIV/AIDS in South India." Aids Care-Psychological and Socio-Medical Aspects of Aids/Hiv_21(4): 473-481.

[43] Sullivan K.M. (2005). Male self-disclosure of HIV positive serostatus to sex partners: a review of the literature. Journal Associated Nurses AIDS Care; 16:33-65.

[44] United Nations General Assembly Special Session (2010) UNGASS Country Progress Report: Nigeria.

[45] Vaux, B. A., Derlega, V. J., Barbee, A. P., Sachdev, M., Antle, B. and Greene, K.(2002). Close relationships as sources of strength or obstacles for mothersncoping with HIV. Journal of Loss and Trauma, 7, 157-184.

[46] Wingood, G. M., Diclemente, R. J., Mikhail, D. H., McCree, S. L., Davies, J. W., Hardin, S.H., Peterson, E. W., Hook N. and Saag M. (2007). "HIV Discrimination and the Health of Women Living with HIV." Women \& Health 46(2-3): 99-112. 